\title{
Clinicopathological factors associated with novel prognostic markers for patients with triple negative breast cancer
}

\author{
Anna M. Badowska-Kozakiewicz ${ }^{1}$, Michał P. Budzik ${ }^{1}$, Anna Liszcz ${ }^{2}$, Maciej T. Sobieraj², \\ Aleksandra I. Czerw ${ }^{3,4}$, Maria Sobol ${ }^{1}$, Janusz Patera ${ }^{5}$, Andrzej Deptała ${ }^{6}$
}

\begin{abstract}
'Department of Biophysics and Human Physiology, Medical University of Warsaw, Warsaw, Poland

'Students' Scientific Organization at the Medical University of Warsaw, Warsaw, Poland ${ }^{3}$ Department of Health Economics and Medical Law, Medical University of Warsaw, Warsaw, Poland

${ }^{4}$ Department of Economic and System Analyses, National Institute of Public Health $\mathrm{NIH}$, Warsaw, Poland

${ }^{5}$ Department of Pathomorphology, Military Institute of Health Services, Warsaw, Poland

${ }^{6}$ Department of Cancer Prevention, Medical University of Warsaw, Warsaw, Poland
\end{abstract}

Submitted: 31 August 2018

Accepted: 4 October 2018

Arch Med Sci 2019: 15 (6): 1433-1442

DOI: https://doi.org/10.5114/aoms.2018.79568

Copyright (c) 2018 Termedia \& Banach

\section{Abstract}

Introduction: Triple negative breast cancer (TNBC) is characterized by a worse prognosis than other breast cancer subtypes. TNBC is defined by lack of expression of estrogen receptor, progesterone receptor and human epidermal growth factor receptor 2 . The aim of this analysis was to evaluate the relationship between immunohistochemical expression of novel prognostic markers (erythropoietin (EPO) and erythropoietin receptor (EPO-R)) and clinicopathological features of TNBC and non-TNBC patients.

Material and methods: Our analysis was conducted on a group of 162 patients with breast carcinoma with lymph node metastasis (111 TNBC and 51 non-TNBC). All statistical analyses were performed with SPSS software $v$ 12.0. Results: Histopathologic subtyping of the 111 triple negative breast cancers identified $89.1 \%$ invasive ductal carcinomas of no special type and $10.9 \%$ other special types of cancers. TNBC more often presented EPO-R and EPO expression (36\%; $37.8 \%)$ than non-TNBC $(23.5 \% ; 29.4 \%)$. Non-TNBC subgroup showed statistically significant correlation only between $\mathrm{Ki}-67$ expression and histological grade $(\mathrm{G} 1-\mathrm{G} 3)(p<0.001)$, while TNBC subgroup demonstrated significant correlation between $\mathrm{Ki}-67$ expression and histological grade (G1-G3) and tumor size (pT1-pT4) as well ( $p=0.002 ; p=0.042)$, between the EPO-R expression and histological grade (G1-G3) $(p<0.001)$.

Conclusions: The relationship between the expression of EPO-R and histological malignancy grade in triple negative breast cancer, suggests that the present EPO-R expression in TNBC may constitute an additional prognostic factor.

Key words: triple negative breast cancer, erythropoietin receptor, erythropoietin, immunohistochemical examination.

\section{Introduction}

Malignant tumors are the second most common cause of death after cardiovascular diseases. The estimates made by epidemiologists indicate that the incidence and death rate for malignant tumors all over the
Corresponding author:

Aleksandra I. Czerw MD, PhD

Department

of Health Economics

and Medical Law

Medical University

of Warsaw

Warsaw, Poland

E-mail: aleksandra.czerw@

wum.edu.pl 
world will probably grow in the coming decades [1]. Among them, breast cancer constitutes a significant diverse group, containing cancers with varying clinical course. Triple negative breast cancer (TNBC) is characterized by a worse prognosis than other breast cancer subtypes. TNBC is defined by lack of expression of estrogen receptor $(E R)$, progesterone receptor (PR) and human epidermal growth factor receptor 2 (HER2) [2]. TNBC is diagnosed in $12-17 \%$ of all breast cancer cases. The risk of this type of cancer is increased before the age of 50 years and is connected with BRCA1 mutation more than any other type. As the TNBC cells are frequently relatively heterogeneous and poorly differentiated, this cancer type is thought to run an aggressive course, and is considered more resistant to chemotherapy and more likely to develop distant metastases. It is also associated with shorter life expectancy and disease-free survival [2, 3].

The term TNBC is sometimes equated to basal-like breast cancer. This is a half-truth, though, for all oncologists must be aware of distinguishing six subtypes of basal type of TNBC, i.e. basal-like 1 $(B L 1)$, basal like 2 (BL2), immunomodulatory (IM), mesenchymal (M), mesenchymal stem-like (MSL) and luminal androgen receptor (LAR). However, BL1 constitutes the great majority among the kind of basal-like breast cancer sensu stricto (81\%); other types possess relevant features only in about $1 / 2$ of all cases (BL2 - 31\%, IM - 58\%, $M-47 \%$ ) or do not express any at all (LAR type, which is considered a luminal A or B). In general, $50 \%$ to $80 \%$ of TNBCs are also basal-like breast cancers $[2,4]$.

A great number of scientific studies have shown that the development of different TNBC forms is closely associated with the induction of various signaling pathways and that TNBC cells show greater sensitivity to different drugs. Hope for improving the results of treatment of TNBC is connected to drugs such as polymerase inhibitors, serine/threonine kinase inhibitors, small molecule HIF- $1 \alpha$ translation inhibitors or EGFR1 inhibitors. Identification of novel TNBC markers provides insight into processes occurring in the tumor, helps to develop new drug combinations and chemotherapeutic strategies and allows one to estimate prognosis. Therefore, it is important to search for new tumor markers and study their relationship with known prognostic factors and predictors, making it possible to understand the TNBC biology. Recent studies showed EPO and EPO-R were strongly correlated with clinicopathological features in many types of cancers. These molecules seem to play a significant role in development of different tumors, with breast cancer among them [2, 4-6].
Apart from its role in erythropoiesis, EPO has a great number of other functions, both in physiological and pathological conditions. It takes part in the cell response to injury, influences the wound healing process, stimulates angiogenesis, and induces proliferation of smooth muscle fibers. Also vasoconstrictive properties of EPO have been described. They allow regulation of blood pressure and blood flow in vessels of the microcirculation. EPO also increases iron absorption from the intestinal tract [7]. Erythropoietin acts on cells by a transmembrane receptor (EPO-R). Although the majority of EPO-R exist on erythroid precursors, their existence has been observed in the brain, myocardium, skeletal muscles, liver, lungs, retina, adrenal glands, parathyroid glands, pancreas, placenta and endothelial cells [4]. EPO and EPO-R are induced by hypoxia in breast cancer and could contribute to increased survival rate of tumor cells via counteraction of hypoxic injury [8].

The aim of this analysis was to evaluate the relationship between immunohistochemical expression of novel prognostic markers (EPO and EPO-R) and clinicopathological features for patients with triple negative breast cancer and non-triple negative breast cancer.

\section{Material and methods}

Studies were conducted in a group of 162 patients with breast carcinoma with lymph node metastasis (111 triple negative breast cancer and 51 non-triple negative breast cancer) in the Department of Pathology, Military Medical Institute in Warsaw. Material for the study came from biopsies, excisional biopsies and modified radical mastectomies. Tumor samples were fixed in $10 \%$ buffered formalin phosphate. After 24-hour fixation, material was dehydrated using alcohol in gradually increasing concentrations and embedded in paraffin. Paraffin blocks were cut into serial sections $4 \mu \mathrm{m}$ in thickness. They were then stained using standard methods. The tumors were classified and graded according to the WHO and the Nottingham modification of the Scarff-Bloom-Richardson systems. In the sections stained with the routine $H \& E$ method, the following determinations were carried out: type of neoplasm (WHO classification), tumor grade including tubule formation, and intensity of division as well as the degree of neoplastic cell differentiation and mitotic index as the mean number of mitotic figures in neoplastic cells counted in 10 fields of vision at 400x magnification (surface area: $0.17 \mathrm{~mm}^{2}$ ).

Paraffin sections on slides covered with $2 \%$ saline solution in acetone at $42^{\circ} \mathrm{C}$ were used for immunohistochemical examination.

Routine tests were performed in order to determine immunohistochemical expression of basic 
profile of diagnostic markers, such as estrogen receptor (ER), progesterone receptor (PR) and HER2. Monoclonal antibodies against receptors for estrogen (Monoclonal Mouse Anti-Human Estrogen Receptor alpha, 1 : 50 dilution, Clone: 1D5, Code: IR654, DAKO, Santa Clara, United States) and progesterone (Monoclonal Mouse Anti-Human Progesterone Receptor, 1 : 400 dilution, Clone: PR636, Code: IR068, DAKO, Santa Clara, United States) were used in order to determine the expression of steroid receptors. Evaluation of the immunohistochemical markers was performed by two pathologists as follows: ER and PR were categorized as negative - $(0 \%)$, low positive - (1-10\%); nuclear staining in $>10 \%$ of tumor cells was considered positive for ER and PR.

The study was conducted as follows: sections were incubated at $60^{\circ} \mathrm{C}$ overnight and subsequently dewaxed. The next step involved revealing the epitope by heating the slides in a buffer for $40 \mathrm{~min}$. Subsequently, preparations were left at room temperature for $20 \mathrm{~min}$. Preparations were rinsed in buffer and endogenous peroxidase was blocked by washing in $3 \% \mathrm{H}_{2} \mathrm{O}_{2}$ for $10 \mathrm{~min}$. In the next step, preparations were incubated with an appropriate antibody for $30 \mathrm{~min}$. After incubation, preparations were rinsed in buffer for $10 \mathrm{~min}$, and then incubated with the reagent (Visualization Reagent) for $30 \mathrm{~min}$. After incubation with the reagent, preparations were washed in TBS (Tris-Buffered Saline, Code: S1968) with pH 7.6 for $10 \mathrm{~min}$, and then incubated with 3,3'-diaminobenzidine (DAB) (Substrate - Chromogen Solution) for 10 min to visualize the color of the reaction. At the end of the procedure, preparations were stained with hematoxylin.

HER2 expression was determined using HercepTest (Code: K5204, DAKO, Santa Clara, United States). It enabled detection of HER2 expression using a polyclonal antibody against this protein (Rb A - Hu HER2 - Rabbit Anti-Human HER2 Protein). Antigen retrieval for HER2 using HercepTest was performed by immersing and incubating the slides in 10-mmol/l citrate buffer in a calibrated water bath $\left(95-99^{\circ} \mathrm{C}\right)$ for $40 \mathrm{~min}( \pm 1 \mathrm{~min})$. After decanting the epitope-retrieving solution, sections were rinsed in the wash buffer and later soaked in the buffer for 5 to $20 \mathrm{~min}$ before staining. The slides were loaded onto the autostainer using the HercepTest program, as described in the manufacturer's insert. In the autostainer, the slides were rinsed, placed in $200 \mu \mathrm{l}$ of peroxidase-blocking reagent for $5 \mathrm{~min}$, rinsed, placed in $200 \mu \mathrm{l}$ of primary anti-HER2 protein (or negative control reagent) for $30 \mathrm{~min}$, rinsed twice and immersed in $200 \mu \mathrm{l}$ of the substrate chromogen solution DAB for $10 \mathrm{~min}$. The slides were counterstained with hematoxylin and finally coverslipped. HER2 results were determined based on the maximum area of staining intensity according to the instructions in the package insert and the ASCO/CAP guidelines as follows: strong, circumferential membranous, staining in $>30 \%$ of invasive carcinoma cell was scored 3+, moderate, circumferential, membranous staining in $\geq 10 \%$ of invasive tumor cells or $3+$ staining in $\leq 30 \%$ of cells was designated as $2+$ staining, weak and incomplete membranous staining in invasive tumor cells was scored $1+$ and no staining was scored 0 . Tumors with 0 and $1+$ staining were considered negative.

A total of 162 cases of breast cancer with metastasis to lymph nodes were assessed for expression of Ki-67 (Monoclonal Mouse Anti-Human Ki-67 1 : 50 dilution, Clone: MIB-5, DAKO, Santa Clara, United States); positivity was quantified as the percentage of positive nuclear staining of the tumor cells, and $10 \%$ was the cut-off point for active proliferation ("> $10 \%$ " and "< $10 \%$ ") $[9,10]$. Immunohistochemistry was performed using EnVision + HRP DakoCytomation (EnVision Dual Link System-HRP, DAB+, Code: K4065, DAKO, Santa Clara, United States).

In all examined breast cancers we also assessed the expression of erythropoietin receptor (EPO-R) using an appropriate antibody against EPO-R antigen (Polyclonal Rabbit Anti-Human EPO-R, 1 : 250 dilution, Clone: C-20, Santa Cruz Biotechnology, Inc., Dallas, United States) and the ImmunoCruz Rabbit ABC Staining System (Santa Cruz Biotechnology, Inc., Dallas, United States) for visualization. EPO-R staining results were scored according to the percentage of membrane positive tumor cells as follows: $(-)<10 \%$; (+), 10-20\%; (++) $>20 \%$. Moderate expression EPO-R was defined as $>20 \%$ tumor cells with positive staining, whereas $<20 \%$ was considered low expression [11]. The immunoexpression of EPO-R was located mainly within cell membranes although in most cases also granular cytoplasmic reaction was observed. Cytoplasmic reaction was considered non-specific and was rejected.

Expression of erythropoietin (EPO) was also assessed in all studied invasive breast cancers through use of an appropriate antibody against EPO antigen (Polyclonal Rabbit Anti-Human EPO, 1 : 100 dilution, Clone: $\mathrm{H}-162$, Santa Cruz Biotechnology, Inc., Dallas, United States) and subsequent application of the ImmunoCruz Rabbit ABC Staining System for visualization (Santa Cruz Biotechnology, Inc., Dallas, United States). EPO staining results were scored according to the percentage of cytoplasmic positive cells as follows: $(-),<10 \%$; (+), 10-20\%; (++), > 20\%. Moderate expression EPO was defined as $>20 \%$ tumor cells with positive staining whereas low expression was $<20 \%$ [12]. For EPO-R and EPO, slides of adult kidney were used as positive controls. 


\section{Statistical analysis}

All statistical analyses were performed with SPSS software $v 12.0$. The $\chi^{2}$ test was used to assess the relationship between EPO, EPO-R, Ki-67 and degree of histological malignancy and clinical staging. Fisher's exact test was used when the expected cell counts were less than 5 . The results were considered as statistically significant if the $p$-value was less than $0.05(p<0.05)$.

\section{Results}

Histopathological examination was performed in tumors obtained from 162 patients suffering from breast cancer. Among 162 breast cancer patients we identified 111 (68.5\%) subjects with triple negative breast cancer (Figures $1 \mathrm{~A}, \mathrm{~B}$ ) (TNBC was identified as ER-negative, PR-negative, and HER2-negative) (Figures $1 \mathrm{C}-\mathrm{F}$ ) and 51 (31.5\%) subjects with non-triple negative breast cancer (Figures 2 A-D). The mean age of patients with TNBC was 47.8 and of patients with non-TNBC was 60.4 years.

Histopathologic subtyping of the 111 triple negative breast cancers identified $89.1 \%$ invasive ductal carcinomas of no special type (IDC-NST) (Figures $1 \mathrm{~A}, \mathrm{~B}$ ) and $10.9 \%$ other special types of cancers: invasive lobular carcinomas, mixed ductal and lobular types, metaplastic carcinomas. All cases of triple negative breast cancer were grouped according to histological grading: 3 (2.7\%) cases were grade 1 (G1), 58 (52.2\%) cases were identified as grade $2(\mathrm{G} 2)$ and $50(45.1 \%)$ cases were grade 3 (G3). Given the histological grade of malignancy, $\mathrm{G} 2$ and $\mathrm{G} 3$ tumors comprised the largest group of triple negative breast cancers.

In our analysis TNBC were most commonly assessed as G2 and G3 (52.2\%; 45.1\%), pT1 and pT2 (34.2\%; 62.1\%), and pN1, pN2 (45\%; 41.4\%). Non-TNBC were most commonly assessed as G2 and G3 (47\%; 47\%), pT1 and pT2 (39.2\%; 47\%) and pN1 (52.9\%). In our analysis a statistically significant association was found only between TNBC and non-TNBC tumor size ( $p T)(p=0.0011)$. Furthermore, in TNBC more commonly than in non-TNBC the presence of necrosis in the tumor mass was observed (36\%; 19.6\%) and a statistically significant correlation between TNBC and non-TNBC in the presence of necrosis was demonstrated $(p=0.036)$.

In all examined breast cancers we also assessed the expression of Ki-67 and novel prognostic markers such as EPO-R and EPO but no statistically significant relationship between TNBC and non-TNBC was revealed. TNBC more often presented EPO-R (Figure $1 \mathrm{G}$ ) and EPO expression (36\%; 37.8\%) than non-TNBC (23.5\%; $29.4 \%)$. In both groups we investigated the correlation between EPO and EPO-R expression and features such as tumor size ( $p T)$, histological grade (G1-G3) and the presence of lymph node metastasis (pN1-pN3). The non-TNBC subgroup showed a statistically significant correlation only between $\mathrm{Ki}-67$ expression and histological grade (G1-G3) $(p<0.001)$, while the TNBC subgroup demonstrated a significant correlation between Ki-67 expression and histological grade (G1-G3) and tumor size (pT1-pT4) as well ( $p=0.002 ; p=0.042$ ) (Figure $1 \mathrm{E})$, between the EPO-R expression and histological grade (G1-G3) $(p<0.001)$. Detailed data and relationships between different parameters are presented in Tables I-V.

\section{Discussion}

Triple negative breast cancer is most commonly found in patients less than 50 years of age [13, 14]. Our analysis also found that TNBC is most common among women before 50 years of age (mean age: 47.8 ).

In our analysis, histopathological subtyping of 111 patients with identified TNBC yielded the following results: $89.1 \%$ IDC-NST and $10.9 \%$ other special types of cancers. Infiltrating ductal carcinoma of no special type (IDC-NST) was the predominant histopathological type. Similar results were obtained by other researchers e.g., Nofech-Mozes et al. (2009), Williams et al. (2009) Atik et al. (2013), Rao et al. (2013), Osman et al. (2014), Sood et al. (2014) and Tawfik et al. (2010) (92\%, 91\%, 27\%, 88\%, 85.7\%, 80.56\% and $81.9 \%$ ), who found that IDC-NST is the dominant histological type in a group of triple negative breast cancers [11, 15-20].

Given the histological grade of malignancy, the largest group of triple negative breast cancers encompassed tumors given $G 2$ and $G 3$ grade. Statistical analysis showed no significant correlation between histological grade (G1-G3) and triple negative tumor morphology $(p>0.05)$. The following authors obtained similar results: Atik et al. (2013) assessing 75\% of cancers in the TNBC group as G3, Carey et al. (2006), who found that in the TNBC group most cases are G3 cancers (26\%) $[16,21]$. In a study on 16 cases of TNBC, Dabbs et al. (2006) found that all tested tumors showed a high degree of histological malignancy [22]. Choi et al. (2012) obtained similar results, stating that in a group of triple negative cancers $63.1 \%$ were G3 tumors [23]. Research by Zhou et al. (2013) also showed that triple negative G2 (51.6\%) and G3 (45.2\%) cancers were most numerous [24]. Osman et al. (2014) confirmed in their study that G3 carcinomas (61.9\%) comprised the largest group of triple negative tumors [18], while Sood and Nigam (2014) pointed to G2 (47.22\%) and G3 (38.89\%) as the most common tumors [19]. 

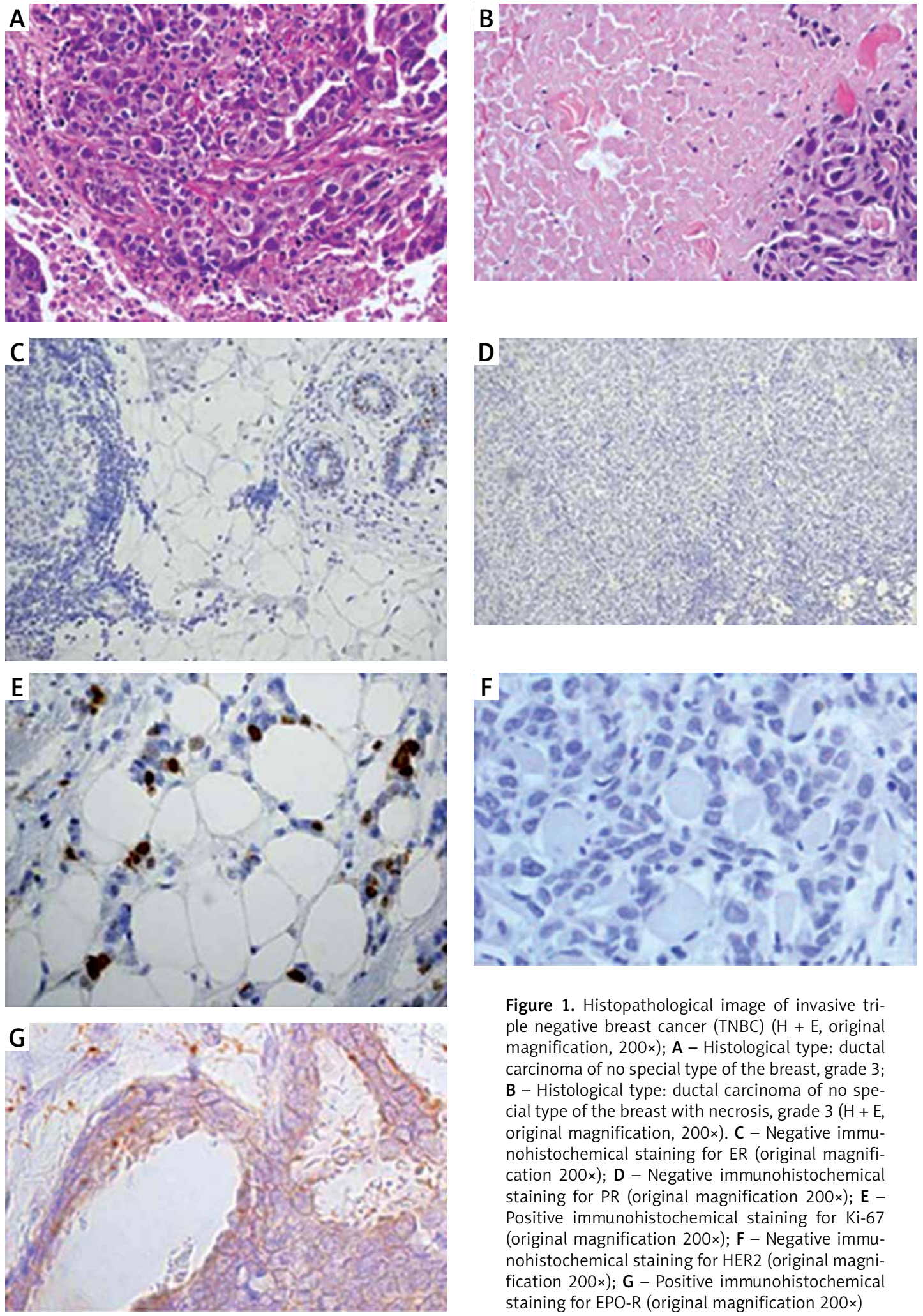

Figure 1. Histopathological image of invasive triple negative breast cancer (TNBC) $(H+E$, original magnification, 200x); A - Histological type: ductal carcinoma of no special type of the breast, grade 3; B - Histological type: ductal carcinoma of no special type of the breast with necrosis, grade $3(\mathrm{H}+\mathrm{E}$, original magnification, 200x). C - Negative immunohistochemical staining for ER (original magnification 200x); D - Negative immunohistochemical staining for PR (original magnification 200x); E Positive immunohistochemical staining for Ki-67 (original magnification 200x); F - Negative immunohistochemical staining for HER2 (original magnification 200x); G - Positive immunohistochemical staining for EPO-R (original magnification 200x)

In our analysis, tumor size was between 2 and $5 \mathrm{~cm}(62.1 \%)$ in the majority of patients, followed by size $<2 \mathrm{~cm}$ (34.1\%). According to studies by Sood and Nigam (2014) in the majority of patients tumor size was between 2.1 and $5 \mathrm{~cm}$ (50\%) followed by < 2 cm (36.11\%) [19]. Rao et al. (2013)

also qualified most of the TNBC tumors as T2 $(2-5 \mathrm{~cm})(68 \%)$ [17].

Our analysis showed expression of $\mathrm{Ki}-67$ (> $10 \%$ ) in $88.2 \%$ of TNBC patients. Sood and Nigam (2014) also noted Ki-67 expression in $80.56 \%$ of TNBCs [19]. Likewise, Rao et al. (2013) 

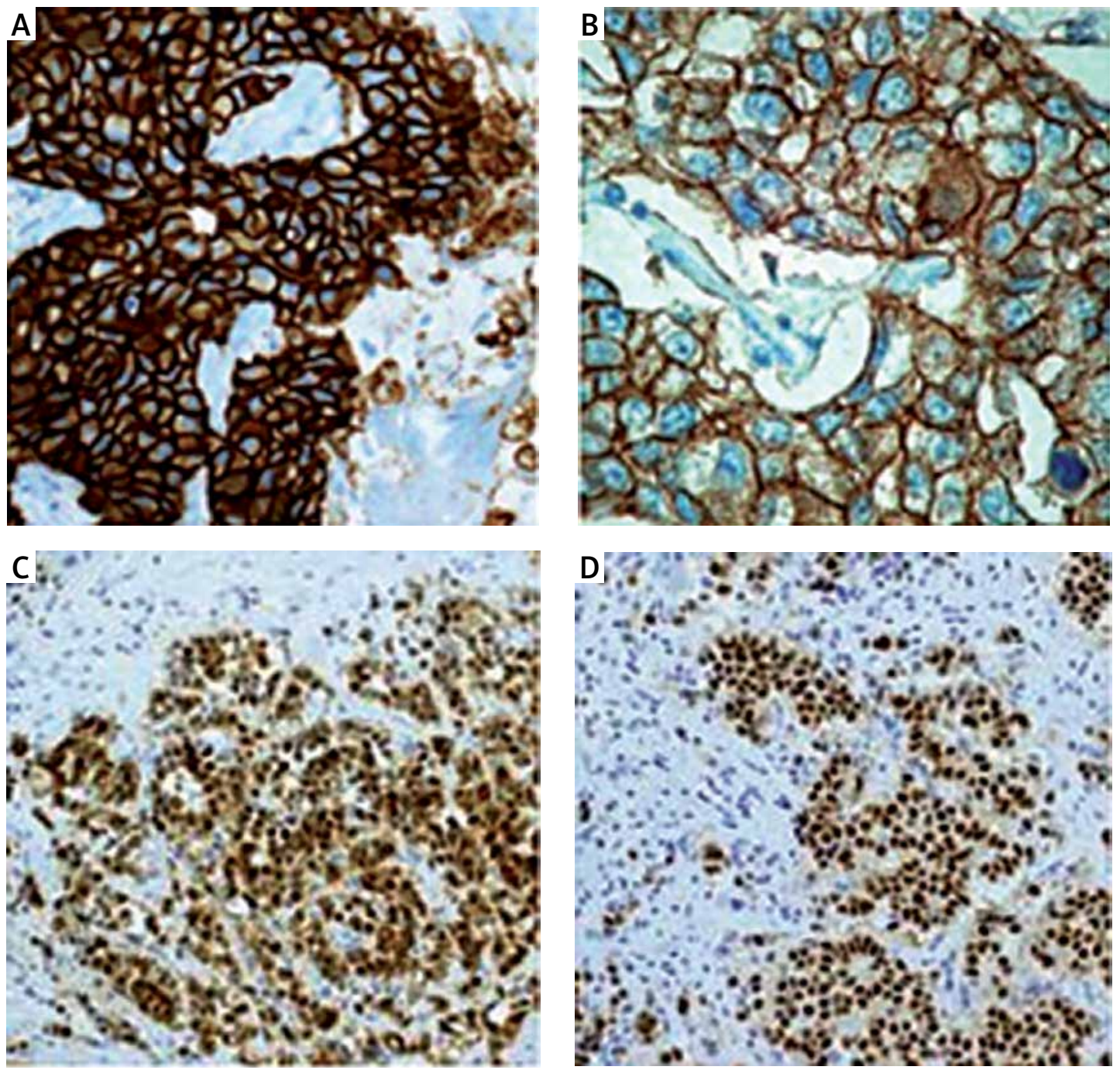

Figure 2. Immunohistochemical image of invasive breast cancer (histological type: ductal carcinoma of no special type of the breast) (non-triple negative breast cancer), A, B - Positive immunohistochemical staining for HER2 (original magnification, 200x); C - Positive immunohistochemical staining for ER (original magnification, 100x); D - Positive immunohistochemical staining for PR (original magnification, 100x)

observed expression of $\mathrm{Ki}-67$ (> 10\%) in $64 \%$ of TNBC patients [17]. Studies conducted by Carey et al. (2006) and Umemura et al. (2005) demonstrated strong immunoreactivity for Ki-67 in triple negative breast carcinoma $[21,25]$. Zhou et al. (2013) found positive expression of Ki-67 in $80.6 \%$ of patients with TNBC [24]. Expression of human Ki-67 protein is closely associated with cell proliferation [26]. Recent data suggest that $\mathrm{Ki}-67$ is a valuable prognostic marker in primary breast cancer and can be used for further classification of TNBCs [26].

We also assessed the proliferative index in TNBC tumors through analysis of Ki-67 expression. In the TNBC group, Ki-67 (> 10\%) expression was noted in the group of cancers characterized by $\mathrm{G} 2$ and $\mathrm{G} 3$ histological grading (49.5\%; $46.5 \%)$, as well as in the group of T2 tumors (66.9\%), and cancers with N2 lymph node status (43.8\%).
There are conflicting reports on the prevalence of lymph node metastases at the time of diagnosis among patients with TNBC. In our analysis we found that women without metastases to regional lymph nodes ( $\mathrm{NNO}$ ) comprised the largest group of all investigated patients with invasive triple negative breast cancer (56.7\%); no statistically significant relationship between lymph node status and histological type of TNBC $(p>0.05)$ was noted. Lymph node status among patients with TNBC was reported as follows: $19.81 \%-N 1,19.81 \%-$ N2, 3.6\% - N3. The analysis also showed no association between tumor size and presence of lymph node metastasis in patients with TNBC, which stood in contradiction to the findings of Thike et al. (2010), who demonstrated a relationship between tumor size and presence of nodal metastases [27]. In studies by Rao et al. (2013) lymph node metastases were found in 37 of 50 patients with TNBC 
(74\% of cases) [17], and TNBC was associated with higher rates of node-positive cases, which was in agreement with the findings of Carey et al. (2006) and Rakha et al. (2007) [21, 28].

In our analysis $30.9 \%$ of all tumors showed central necrosis. In TNBC more commonly than in non-TNBC the presence of necrosis was observed (36\%; 19.6\%). Yehia et al. (2015) in their study divided breast cancers into three subgroups (TNBC, HER2+ and ER+/PR+). $15.3 \%$ of all tumors showed central fibrosis and tumor necrosis, which differed significantly among the three groups ( $p=0.019)$. TNBC had the highest values among all groups even after adjusting the results for age. Necrosis was observed in $25.8 \%$ of TNBC, $9.4 \%$ of HER2+ and $10.9 \%$ of ER+/PR+ cancers [29].

Currently there are no representative research results on EPO and EPO-R expression in triple negative breast cancer. Our analysis appears to be the first to evaluate expression of these novel prognostic markers with particular emphasis on TNBC and its comparison with other molecular subtypes of breast cancer. As shown in Table II, TNBC more often presented EPO-R and EPO expression (36\%; $37.8 \%)$ than non-TNBC (23.5\%; $29.4 \%)$. Also worth highlighting is the fact that the TNBC subgroup, but not the non-TNBC subgroup, demonstrated a significant correlation between EPO-R expression and histological grade (G1-G3) $(p<0.001)$, as presented in Tables IV and V.

Despite the fact that EPO originally had been given only a role in the regulation of erythropoiesis, this protein turned out to be an important link of many signaling pathways, both in multiple normal and cancerous nonhematopoietic tissues. It seems very likely that enhanced expression of the EPO receptor in tumor cells is inversely proportional to its degree of differentiation. In recent years functional autocrine/paracrine EPO/EPO-R systems have been described in human cancer cells originating from breast cancer, cervical cancer, melanoma and prostate cancer. These data suggest that the EPO/EPO-R axis may affect tumor growth, progression and metastasis [30-35].

Isolated expression of EPO-R is further demonstrated on the cell surface of malignant tumors such as breast cancer, prostate cancer, squamous cell cancers, multiple myeloma and their metastases [36]. The presence of EPO-R sensitizes these tissues to the effect of systemic endogenous erythropoietin, although we cannot exclude signals from paracrine pathways as it is often the case that EPO is produced by healthy tissues surrounding the tumor or other adjacent tissues.

Larsson et al. (2009) described expression of EPO and EPO-R in the cell lines and breast cancer tissue samples, with particular emphasis on
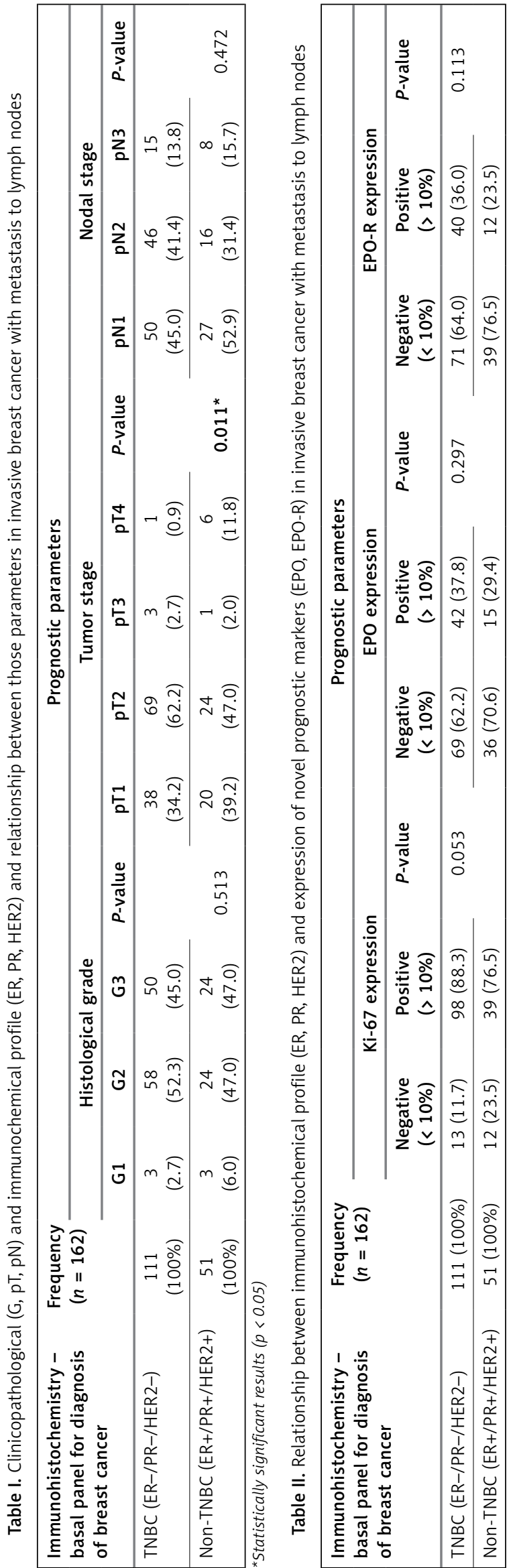
Table III. Analysis of relations between immunohistochemical profile (ER, PR, HER2) and pathological parameters in invasive breast cancer with metastasis to lymph nodes

\begin{tabular}{|c|c|c|c|c|c|c|c|}
\hline \multirow[t]{3}{*}{ Immunohistochemistry } & \multirow{3}{*}{$\begin{array}{l}\text { Frequency } \\
(n=162)\end{array}$} & \multicolumn{6}{|c|}{ Prognostic parameters } \\
\hline & & \multicolumn{3}{|c|}{ Tumor necrosis } & \multicolumn{3}{|c|}{$\begin{array}{l}\text { Histological type of invasive breast } \\
\text { cancer }\end{array}$} \\
\hline & & Positive & Negative & $P$-value & IDC-NST & $\begin{array}{l}\text { Other } \\
\text { types }\end{array}$ & $P$-value \\
\hline TNBC & $111(100 \%)$ & $40(36.0)$ & $71(64.0)$ & \multirow[t]{2}{*}{$0.036^{*}$} & $99(89.1)$ & $12(10.9)$ & \multirow[t]{2}{*}{0.858} \\
\hline Non-TNBC & $51(100 \%)$ & $10(19.6)$ & $41(80.4)$ & & $45(88.2)$ & $6(11.8)$ & \\
\hline
\end{tabular}

*Statistically significant results $(p<0.05)$.

Table IV. Relationship between clinicopathological features of non-TNBC and expression of novel prognostic markers (EPO, EPO-R) in invasive breast cancer with metastasis to lymph nodes

\begin{tabular}{|c|c|c|c|c|c|c|c|c|c|c|}
\hline \multirow{2}{*}{\multicolumn{2}{|c|}{$\begin{array}{l}\text { Clinicopathological } \\
\text { features } \\
\text { non-TNBC }\end{array}$}} & \multicolumn{9}{|c|}{ Prognostic parameters } \\
\hline & & \multicolumn{3}{|c|}{ Ki-67 expression } & \multicolumn{3}{|c|}{ EPO expression } & \multicolumn{3}{|c|}{ EPO-R expression } \\
\hline & & $\begin{array}{c}\text { Negative } \\
(<10 \%)\end{array}$ & $\begin{array}{l}\text { Positive } \\
\text { (> 10\%) }\end{array}$ & $P$-value & $\begin{array}{c}\text { Negative } \\
(<10 \%)\end{array}$ & $\begin{array}{l}\text { Positive } \\
\text { (> 10\%) }\end{array}$ & $P$-value & $\begin{array}{l}\text { Negative } \\
(<10 \%)\end{array}$ & $\begin{array}{l}\text { Positive } \\
\text { (> 10\%) }\end{array}$ & $P$-value \\
\hline \multirow{3}{*}{$\begin{array}{l}\text { Histological } \\
\text { grade }\end{array}$} & G1 & 3 & 0 & $<0.001$ & 1 & 2 & 0.088 & 1 & 2 & 0.247 \\
\hline & $\mathrm{G} 2$ & 9 & 15 & & 20 & 4 & & 19 & 5 & \\
\hline & G3 & 0 & 24 & & 15 & 9 & & 19 & 5 & \\
\hline \multirow[t]{4}{*}{ Tumor stage } & pT1 & 8 & 12 & 0.127 & 14 & 6 & 0.564 & 16 & 4 & 0.415 \\
\hline & pT2 & 4 & 20 & & 17 & 7 & & 18 & 6 & \\
\hline & pT3 & 0 & 1 & & 0 & 1 & & 0 & 1 & \\
\hline & pT4 & 0 & 6 & & 5 & 1 & & 5 & 1 & \\
\hline \multirow[t]{3}{*}{ Nodal stage } & pN1 & 9 & 18 & 0.168 & 20 & 7 & 0.101 & 21 & 6 & 0.112 \\
\hline & pN2 & 3 & 13 & & 10 & 6 & & 8 & 8 & \\
\hline & pN3 & 0 & 8 & & 6 & 2 & & 7 & 1 & \\
\hline
\end{tabular}

${ }^{*}$ Statistically significant results $(p<0.05)$.

Table V. Relationship between clinicopathological features of TNBC and expression of novel prognostic markers (EPO, EPO-R) in invasive breast cancer with metastasis to lymph nodes

\begin{tabular}{|c|c|c|c|c|c|c|c|c|c|c|}
\hline \multirow{2}{*}{\multicolumn{2}{|c|}{$\begin{array}{l}\text { Clinicopathological } \\
\text { features } \\
\text { TNBC }\end{array}$}} & \multicolumn{9}{|c|}{ Prognostic parameters } \\
\hline & & \multicolumn{3}{|c|}{ Ki-67 expression } & \multicolumn{3}{|c|}{ EPO expression } & \multicolumn{3}{|c|}{ EPO-R expression } \\
\hline & & $\begin{array}{l}\text { Negative } \\
(<10 \%)\end{array}$ & $\begin{array}{l}\text { Positive } \\
\text { (>10\%) }\end{array}$ & $P$-value & $\begin{array}{l}\text { Negative } \\
(<10 \%)\end{array}$ & $\begin{array}{l}\text { Positive } \\
\text { (>10\%) }\end{array}$ & $P$-value & $\begin{array}{l}\text { Negative } \\
(<10 \%)\end{array}$ & $\begin{array}{l}\text { Positive } \\
\text { (>10\%) }\end{array}$ & $P$-value \\
\hline \multirow{3}{*}{$\begin{array}{l}\text { Histological } \\
\text { grade }\end{array}$} & G1 & 3 & 0 & 0.002 & 0 & 3 & 0.087 & 0 & 3 & $<0.001$ \\
\hline & $\mathrm{G} 2$ & 10 & 48 & & 38 & 20 & & 30 & 28 & \\
\hline & G3 & 0 & 50 & & 31 & 19 & & 41 & 9 & \\
\hline \multirow[t]{4}{*}{ Tumor stage } & pT1 & 9 & 29 & 0.042 & 22 & 16 & 0.236 & 28 & 10 & 0.138 \\
\hline & pT2 & 4 & 65 & & 44 & 25 & & 42 & 27 & \\
\hline & pT3 & 0 & 3 & & 2 & 1 & & 1 & 2 & \\
\hline & pT4 & 0 & 1 & & 1 & 0 & & 0 & 1 & \\
\hline \multirow[t]{3}{*}{ Nodal stage } & pN1 & 10 & 40 & 0.054 & 29 & 21 & 0.627 & 37 & 13 & 0.134 \\
\hline & pN2 & 3 & 43 & & 31 & 15 & & 26 & 20 & \\
\hline & pN3 & 0 & 15 & & 9 & 6 & & 8 & 7 & \\
\hline
\end{tabular}


patients treated with the antiestrogenic drug tamoxifen. All tested samples showed expression of both proteins. EPO and EPO-R expression was also observed in normal healthy breast tissue. Tamoxifen treatment significantly increased recurrence-free survival (RFS) in patients with $\mathrm{ER}^{+} /$ $\mathrm{PR}^{+}$tumors with low EPO-R expression but had no effect on RFS in patients with high EPO-R expression. The finding that EPO-R gives prognostic information in $\mathrm{ER}^{+}$but not in ER- breast cancers has to be further elucidated but can support view that EPO-R has a specific role associated with estrogen receptor [37].

Acs et al. (2002) investigated the EPO and EPO-R expression in breast tumor cells. Benign cancer cells showed weak to moderate EPO and EPO-R expression. EPO-R expression in malignant breast cancer measured by immunohistochemistry was clearly higher than in benign tumor cells. The most notable EPO expression was observed in tumor cells adjacent to the areas of necrosis and cells in the periphery of the lesion, especially in places most infiltrating surrounding tissues. It was also observed that EPO-R expression was greater in highly differentiated tumors, tumors with presence of necrotic areas, invasion of lymph vessels, metastasis to lymph nodes and those with loss of expression of hormone receptors (ER, PR). Induction of the erythropoietin signaling pathway represents a mechanism wherein local hypoxia induces carcinogenesis [38].

In conclusion, we found that there is a significant link between EPO-R and the histological grade of TNBC. In effect, the expression of this marker in tumor cells may be considered an additional prognostic factor. However, many more prospective studies based on larger groups of TNBC patients need to be performed in order to sufficiently establish the importance and clinicopathological meaning of this dependence.

The relationship between the expression of EPO-R and histological malignancy grade in triple negative breast cancer (TNBC) suggests that EPO-R expression in TNBC may constitute an additional prognostic factor.

\section{Conflict of interest}

The authors declare no conflict of interest.

\section{References}

1. Augustynowicz A, Czerw Al, Deptała A. Health needs as a priority of local authorities in Poland based on the example of implementation of health policy cancer programmes. Arch Med Sci 2018; 14: 1439-49.

2. Badve S, Dabbas DJ, Schnitt SJ, et al. Basal-like and triple negative breast cancers: a critical review with an emphasis on the implications for pathologists and oncologists. Mod Pathol 2011; 24: 157-67.
3. Kołacińska A, Chałubińska J, Błasińska-Morawiec M, et al. Pathological complete response in younger and older breast cancer patients. Arch Med Sci 2012; 8: 310-5.

4. Cheang MC, Voduc D, Bajdik C, et al. Basal-like breast cancer defined by five biomarkers has superior prognostic value than triple-negative phenotype. Clin Cancer Res 2008; 14: 1368-76.

5. Kutomi G, Ohmura T, Suzuki Y, et al. Clinicopathological characteristics of basal type breast cancer in triple - negative breast cancer. J Cancer Therapy 2012; 3: 836-40.

6. Nielsen TO, Hsu FD, Jensen K, et al. Immunohistochemical and clinical characterization of the basal-like subtype of invasive breast carcinoma. Clin Cancer Res 2004; 10: 5367-74.

7. Arcasoy MO, Amin K, Vollmer RT, JiangX, Demark-Wahnefried W, Haroon ZA. Erythropoietin and erythropoietin receptor expression in human prostate cancer. Mod Pathol 2005; 18: 421-30.

8. Acs G, Acs P, Beckwith SM, et al. Erythropoietin and erythropoietin receptor expression in human cancer. Cancer Res 2001; 61: 3561-5.

9. Keam B, Im S, Lee K, et al. Ki-67 can be used for further classification of triple-negative breast cancer into two subtypes with different response and prognostic. Breast Cancer Res 2011; 13: R22.

10. Gutierrez C, Schiff R. HER 2 Biology, detection, and clinical implications. Arch Pathol Lab Med 2011; 135: 907-22.

11. Nofech-Mozes S, Trudeau M, Kahn HK, et al. Patterns of recurrence in the basal and non-basal subtypes of triple - negative breast cancers. Breast Cancer Res Treat 2009; 118: 131-7.

12. Lester SC, Bose S, Chen YY, et al. Protocol for the examination of specimens with invasive carcinoma of the breast. Arch Pathol Lab Med 2009; 133: 1515-38.

13. Thike AA, Cheok PY, Jara-Lazaro AR, Tan B, Tan P, Tan PH. Triple-negative breast cancer: clinicopathological characteristics and relationship with basal-like breast cancer. Mod Pathol 2010; 23: 123-33.

14. Wong CS, Chow KY, Lim GH, Bhalla V, Lee HP, Chia KS. Cancer Survival in Singapore 1968-2002. Singapore Cancer Registry 2008.

15. Williams DJ, Cohen J, To TV, et al. Triple negative breast carcinoma in women from Vietnam and United States: characterization of differential markers expression by tissue microarray. Hum Pathol 2009; 40: 1176-81.

16. Atik E, Guray M, Ozgur T, Canda T. Characterization of immunohistochemical markers in triple negative breast carcinomas. JBUON 2013; 18: 886-90.

17. Rao C, Shetty J, Krishan Prasad HL. Immunohistochemical profile and morphology in triple-negative breast cancers. J Clin Diagn Res 2013; 7: 1361-5.

18. Osman NM, Chalabi N, Raboh NMA. Triple negative breast cancer: MRI features in comparison to other breast cancer subtypes with correlation to prognostic pathologic factors. Egypt J Radiol Nucl Med 2014; 45: 1309-16.

19. Sood N, Nigam JS. Correlation of CK5 and EGFR with clinicopathological profile of triple-negative breast cancer. Pathol Res Inter 2014; 2014: 141864.

20. Tawfik O, Davis K, Kimler BF, et al. Clinicopathological characteristics of triple negative invasive mammary carcinomas in African-American versus Caucasian women. Ann Clin Lab Sci 2010; 40: 315-23.

21. Carey LA, Perou CM, Livasy CA, et al. Race, breast cancer subtypes, and survival in the Carolina Breast Cancer Study. JAMA 2006; 295: 2492-502. 
22. Dabbs DJ, Chivukula M, Carter G, Bhargava R. Basal phenotype of ductal carcinoma in situ: recognition and immunohistologic profile. Mod Pathol 2006; 19: 1506-11.

23. Choi J, Jubg WH, Koo JS. Clinicopathologic features of molecular subtypes of triple negative breast cancer based on immunohistochemical markers. Histol Histopathol 2012; 27: 1481-93.

24. Zhou L, Li K, Luo Y, et al. Novel prognostic markers for patients with triple - negative breast cancer. Hum Pathol 2013; 44: 2180-7.

25. Umemura S, Takekoshi S, Suzuki Y, Saitoh Y, Tokuda Y, Osamura RY. Estrogen receptor negative and human epidermal growth factor receptor 2 negative breast cancer tissue has the highest Ki-67 labeling index and EGFR expression. Oncol Rep 2005; 14: 337-43.

26. Yerushalami R, Woods R, Ravdin PM, Hayes MM, Gelmon KA. Ki-67 in breast cancer: prognostic and predictive potential. Lancet Oncol 2010; 11: 174-83.

27. Thike AA, Iqbal J, Cheok PY, et al. Triple negative breast cancer: outcome correlation with immunohistochemical detection of basal markers. Am J Surg Pathol 2010; 34: 956-64.

28. Rakha EA, El-Sayed ME, Green AR, Lee AH, Robertson JF, Ellis IO. Prognostic markers in triple-negative breast cancer. Cancer 2007; 109: 25-32.

29. Yehia L, Boulos F, Jabbour M, et al. Expression of HIF-1alpha and markers of angiogenesis are not significantly different in triple negative breast cancer compared to other breast cancer molecular subtypes: implications for future therapy. PLoS One 2015; 10: e0129356.

30. Acs G, Chen M, Xu X, Acs P, Verma A, Koch CJ. Autocrine erythropoietin signaling inhibits hypoxia-induced apoptosis in human breast carcinoma cells. Cancer Lett 2004; 214: 243-51.

31. Trost N, Stepisnik T, Berne S, et al. Recombinant hu man erythropoietin alters gene expression and stimulates proliferation of MCF-7 breast cancercells. Radiol Oncol 2013; 47: 382-9.

32. Kumar SM, Acs G, Fang D, Herlyn M, Elder DE, Xu X Functional erythropoietin autocrine loop in melanoma. Am J Pathol 2005; 166: 823-30.

33. Lopez TV, Lappin TR, Maxwell P, et al. Autocrine/paracrine erythropoietin signalling promotes JAK/STAT-dependent proliferation of human cervical cancer cells. Int J Cancer 2011; 129: 2566-76.

34. Jeong JY, Hoxhaj G, Socha AL, Sytkowski AJ, Feldman L. An erythropoietin autocrine/paracrine axis modulates the growth and survival of human prostate cancer cells. Mol Cancer Res 2009; 7: 1150-7.

35. Pelekanou V, Kampa M, Kafousi M, et al. Erythropoietin and its receptor in breast cancer: correlation with steroid receptors and outcome. Cancer Epidemiol Biomarkers Prev 2007; 16: 2016-23.

36. Lai SY, Grandis JR. Understanding the presence and function of erythropoietin receptors on cancer cells. J Clin Oncol 2006; 24: 4675-6.

37. Larsson AM, Jirström K, Fredlund E, et al. Erythropoietin receptor expression and correlation to tamoxifen response and prognosis in breast cancer. Clin Cancer Res 2009; 15: 5552-9.

38. Acs G, Zhang PJ, Rebbeck TR, Acs P, Verma A. Immunohistochemical expression of erythropoietin receptor in breast carcinoma. Cancer 2002; 95: 969-81. 